OPEN ACCESS

Edited by:

Ferdinand X. Frauscher,

Innsbruck Medical University, Austria

Reviewed by:

Juan Gomez Rivas,

Hospital Clínico San Carlos, Spain

Giovanni Battista Di Pierro,

Sapienza University of Rome, Italy

${ }^{*}$ Correspondence:

Mike Wenzel

mike.wenze/@kgu.de

Specialty section:

This article was submitted to

Genitourinary Surgery,

a section of the journal

Frontiers in Surgery

Received: 15 August 2021

Accepted: 25 October 2021

Published: 09 December 2021

Citation

Wenzel $M$, Preisser $F$, Hoeh $B$ Welte MN, Humke $C$, Wittler $C$,

Würnschimmel C, Becker A, Karakiewicz PI, Chun FKH, Mandel $P$

and Kluth LA (2021) Influence of

Biopsy Gleason Score on the Risk of Lymph Node Invasion in Patients With Intermediate-Risk Prostate Cancer Undergoing Radical Prostatectomy.

Front. Surg. 8:759070

doi: 10.3389/fsurg.2021.759070

\section{Influence of Biopsy Gleason Score on the Risk of Lymph Node Invasion in Patients With Intermediate-Risk Prostate Cancer Undergoing Radical Prostatectomy}

\author{
Mike Wenzel ${ }^{1,2 *}$, Felix Preisser ${ }^{1}$, Benedikt Hoeh ${ }^{1}$, Maria N. Welte ${ }^{1}$, Clara Humke ${ }^{1}$, \\ Clarissa Wittler ${ }^{1}$, Christoph Würnschimmel ${ }^{2,3}$, Andreas Becker ${ }^{1}$, Pierre I. Karakiewicz ${ }^{2}$, \\ Felix K. H. Chun ${ }^{1}$, Philipp Mandel ${ }^{1}$ and Luis A. Kluth ${ }^{1}$

\begin{abstract}
${ }^{1}$ Department of Urology, University Hospital Frankfurt, Frankfurt, Germany, ${ }^{2}$ Cancer Prognostics and Health Outcomes Unit, Division of Urology, University of Montreal Health Center, Montreal, QC, Canada, ${ }^{3}$ Martini-Klinik Prostate Cancer Center, University Hospital Hamburg-Eppendorf, Hamburg, Germany
\end{abstract}

Objective: To analyze the influence of biopsy Gleason score on the risk for lymph node invasion $(\mathrm{LNI})$ during pelvic lymph node dissection $(\mathrm{PLND})$ in patients undergoing radical prostatectomy (RP) for intermediate-risk prostate cancer (PCa).

Materials and Methods: We retrospectively analyzed 684 patients, who underwent RP between 2014 and June 2020 due to PCa. Univariable and multivariable logistic regression, as well as binary regression tree models were used to assess the risk of positive LNI and evaluate the need of PLND in men with intermediate-risk PCa.

Results: Of the 672 eligible patients with RP, 80 (11.9\%) men harbored low-risk, $32(4.8 \%)$ intermediate-risk with international society of urologic pathologists grade (ISUP) 1 (IR-ISUP1), 215 (32.0\%) intermediate-risk with ISUP 2 (IR-ISUP2), 99 (14.7\%) intermediate-risk with ISUP 3 (IR-ISUP3), and 246 (36.6\%) high-risk PCa. Proportions of LNI were 0, 3.1, 3.7, 5.1, and 24.0\% for low-risk, IR-ISUP1, IR-ISUP 2, IR-ISUP-3, and high-risk PCa, respectively $(p<0.001)$. In multivariable analyses, after adjustment for patient and surgical characteristics, IR-ISUP1 [hazard ratio (HR) 0.10, $p=0.03$ ], IRISUP2 (HR 0.09, $p<0.001$ ), and IR-ISUP3 (HR 0.18, $p<0.001$ ) were independent predictors for lower risk of $\mathrm{LNI}$, compared with men with high-risk $\mathrm{PCa}$ disease.

Conclusions: The international society of urologic pathologists grade significantly influence the risk of $L N I$ in patients with intermediate- risk PCa. The risk of $L N I$ only exceeds 5\% in men with IR-ISUP3 PCa. In consequence, the need for PLND in selected patients with IR-ISUP 1 or IR-ISUP2 PCa should be critically discussed.

Keywords: prostate cancer, lymph node dissection, intermediate risk, Gleason score, lymph node metastases, predictor 


\section{INTRODUCTION}

Prostate cancer $(\mathrm{PCa})$ is the most common malignancy in men and still results in high amounts of cancer-specific deaths (1-5) worldwide. There is an ongoing debate, which patients undergoing radical prostatectomy (RP) in a curative intent, benefit most from pelvic lymph node dissection (PLND) $(6,7)$. Due to the morbidity caused by PLND $(8,9)$, European guidelines recommend PLND in selected patients with a risk for lymph node invasion (LNI) of $>5 \%$, using specific nomograms (1014). Temporal trends have shown higher rates of PLND in patients with D'Amico intermediate and high risk in recent years (15). Nonetheless, since patients with D'Amico intermediaterisk group PCa have a high heterogeneity according to tumor characteristics (16-18), it still remains unclear, if PLND can be avoided in selected patients with intermediate-risk PCa (19).

We tried to address this relevant question by analyzing patients, who underwent RP with PLND for intermediaterisk $\mathrm{PCa}$. In the present study, we stratified patients with intermediate-risk PCa by their international society of urologic pathologists (ISUP) grade, to identify patients with PCa with higher risk of LNI and the need of undergoing PLND.

\section{MATERIALS AND METHODS}

\section{Study Population}

After approval of the ethic committee, 684 consecutive patients who underwent RP (either robotic or open) at the Department of Urology at Frankfurt University Hospital between January 2014 to June 2020 were identified from the institutional database and evaluated retrospectively. Indications for RP was biopsy (either systematic or targeted biopsy) confirmed PCa. Patients with unknown PSA at PCa diagnosis, unknown clinical T stage and unknown ISUP grade at biopsy were excluded $(n=12)$. This selection criteria yielded in 672 eligible patients, of whom 366 patients harbored intermediate-risk $\mathrm{PCa}$.

\section{Statistical Analysis}

Descriptive statistics included frequencies and proportions for categorical variables. The means, medians and interquartile ranges (IQR) were reported for continuously coded variables. The Chi-square test was used for statistical significance in proportions' differences. The $t$-test and Kruskal-Wallistest examined the statistical significance of means' and distributions' differences.

To investigate the effect of Gleason score at biopsy on LNI, intermediate-risk subgroups were stratified into intermediate-risk ISUP 1, intermediate-risk ISUP 2, and intermediate-risk ISUP 3. LNI represented the clinical endpoint of this study. Univariable and multivariable logistic regression models (after adjustment for patient and surgical characteristics such as age, prostate volume, body mass index, and number of removed lymph nodes) were fitted to predict LNI in ISUP subgroups of patients with intermediate-risk PCa. Moreover, we compared the rates of LNI within the intermediate-risk subgroups and relative to patients with low-risk and high-risk PCa. Finally, D'Amico risk groups and ISUP grade at biopsy were used to predict LNI with a binary regression tree. All tests were two sided with a level of significance set at $p<0.05$, and $\mathrm{R}$ software environment for statistical computing and graphics (version 3.4.3) was used for all analyses.

\section{RESULTS}

\section{Descriptive Characteristics of the Study Population}

Of the 672 eligible patients with RP (Table 1), patients with low risk accounted for 80 (11.9\%), intermediate-risk ISUP 1 (IRISUP1) for 32 (4.8\%), intermediate-risk ISUP 2 (IR-ISUP2) for 215 (32.0\%), intermediate-risk ISUP 3 (IR-ISUP3) for 99 (14.7\%), and high risk for 246 (36.6\%). The median age was lowest in patients with low risk (63 years), followed by IR-ISUP2 (66 years), IR-ISUP3 (66 years), IR-ISUP1 (67 years), and high risk (67 years), respectively ( $p<0.01$ ). The median PSA was lowest in men with low-risk PCa $(6.4 \mathrm{ng} / \mathrm{ml})$, followed by IR-ISUP2 $(6.6 \mathrm{ng} / \mathrm{ml})$, IR-ISUP3 $(7.5 \mathrm{ng} / \mathrm{ml})$, high risk $(11.1 \mathrm{ng} / \mathrm{ml})$, and IR-ISUP1 $(11.5 \mathrm{ng} / \mathrm{ml})$, in that order $(p<0.001)$.

Within the intermediate-risk patient cohort, cT1c and cT2b stages were highest in IR-ISUP1 cohort (62.5 and 31.2\%), followed by IR-ISUP2 (60.5 and 11.2\%) and IR-ISUP3 (50.5 and $9.1 \%)$. Conversely, cT2a stage was lowest in IR-ISUP1 $(6.2 \%)$, relative to IR-ISUP2 (28.4\%), and IR-ISUP3 (40.4\%). Moreover, proportions of pathological ISUP score $2-5$ and locally advanced pT3-4 stages after RP were significantly higher in both IR-ISUP2 and IR-ISUP3, compared with IR-ISUP1 (all $p<0.05$ ).

\section{Influence of ISUP Grade in Patients With Intermediate-Risk PCa on the Risk of LNI}

Pelvic lymph node dissection was performed in 78.8, 93.8, 95.8, 98.0, and $97.2 \%$ of patients with low-risk, IR-ISUP1, IR-ISUP2, IR-ISUP3, and high-risk PCa. The median number of removed lymph nodes during PLND was 8 (IQR 5-12), 10 (IQR 5-15), 12 (IQR 8-17), 12 (IQR 7-12), and 15 (IQR 9-21) in patients with low-risk, IR-ISUP1, IR-ISUP2, IR-ISUP3, and high-risk PCa, respectively $(p=0.8)$. The risk of LNI differed significantly across the analyzed subgroups with, respectively, $0,3.1,3.7,5.1$, and $24.0 \%$ for patients with low-risk, IR-ISUP1, IR-ISUP2, IR-ISUP3, and high-risk PCa $(p<0.001)$. The median number of positive lymph nodes was 0 (IQR 0-0), 1 (IQR 1-1), 1 (IQR 1-3), 1 (IQR 1-2), and 2 (IQR 1-4) for patients with low-risk, IR-ISUP1, IRISUP2, IR-ISUP3, and high-risk PCa. In binary regression tree models (Figure 1), stratification firstly according to D'Amico risk group and secondly according to ISUP grade/Gleason score at biopsy predicted probabilities of LNI with an accuracy of 0.753 .

In multivariable analyses (Table 2) after adjustment for patient and surgical characteristics (age, prostate volume, body mass index, and number of removed lymph nodes), IR-ISUP1 [hazard ratio (HR) 0.10 , CI $0.01-0.49, p=0.03$ ], IR-ISUP2 (HR 0.09 , CI $0.04-0.21, p<0.001$ ), and IR-ISUP3 (HR 0.18, CI 0.06$0.42, p<0.001)$ were independent predictors of lower risk of LNI, compared to patients with high-risk PCa. Moreover, number of removed lymph nodes was a predictor for LNI in univariable model, albeit not reaching significance in multivariable analyses (HR 1.03, CI 1.00-1.06, $p=0.058$ ). 
TABLE 1 | Descriptive characteristics of 672 patients, who underwent radical prostatectomy, according to D'Amico risk score and also according to ISUP grade in intermediate risk prostate cancer.

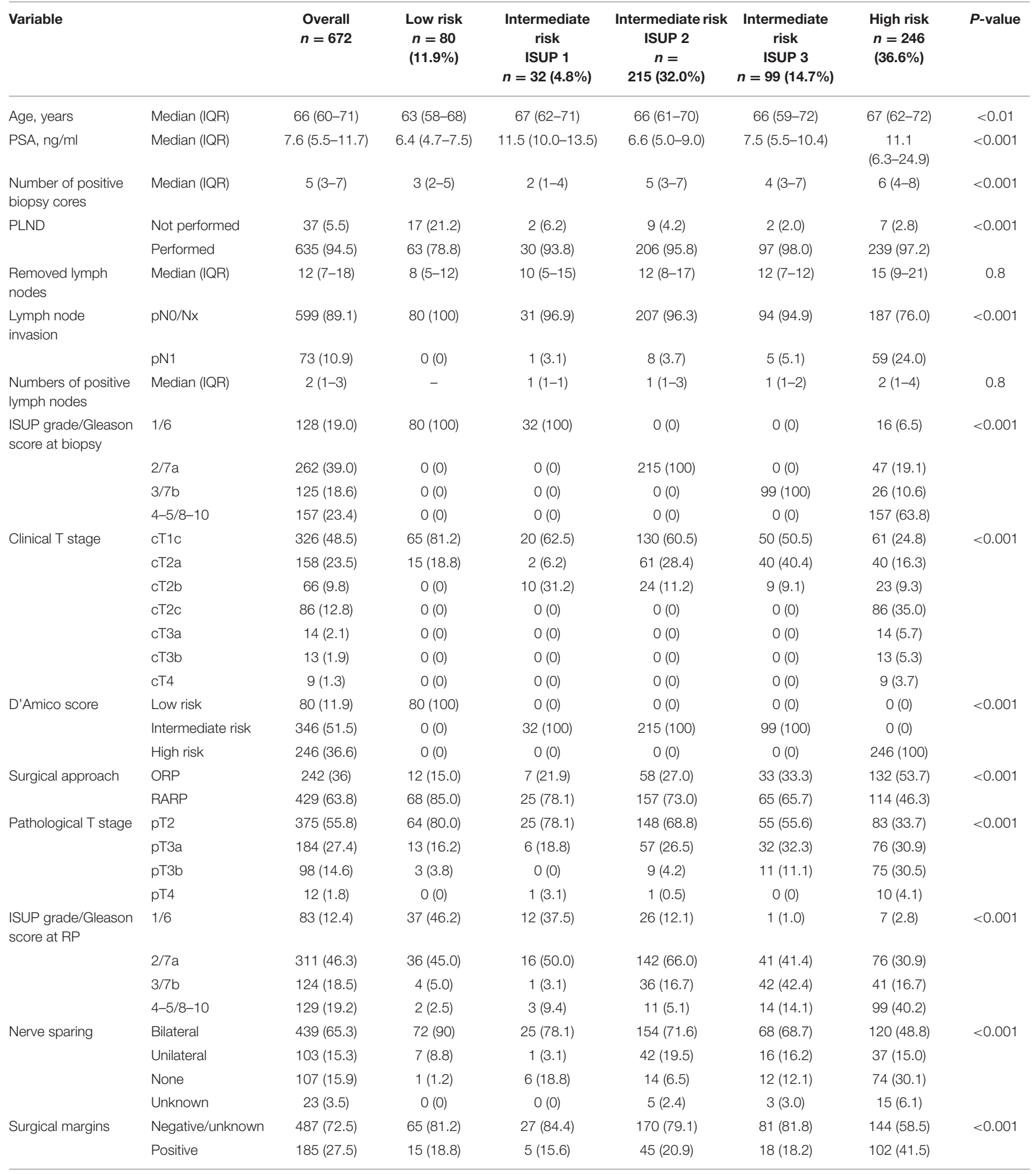

ISUP, International society of urological pathologists grade; GS, Gleason score; PSA, Prostate Specific Antigen; PLND, Pelvic lymph node dissection; ORP, Open Radical Prostatectomy; RARP, Robot-assisted Radical Prostatectomy. 


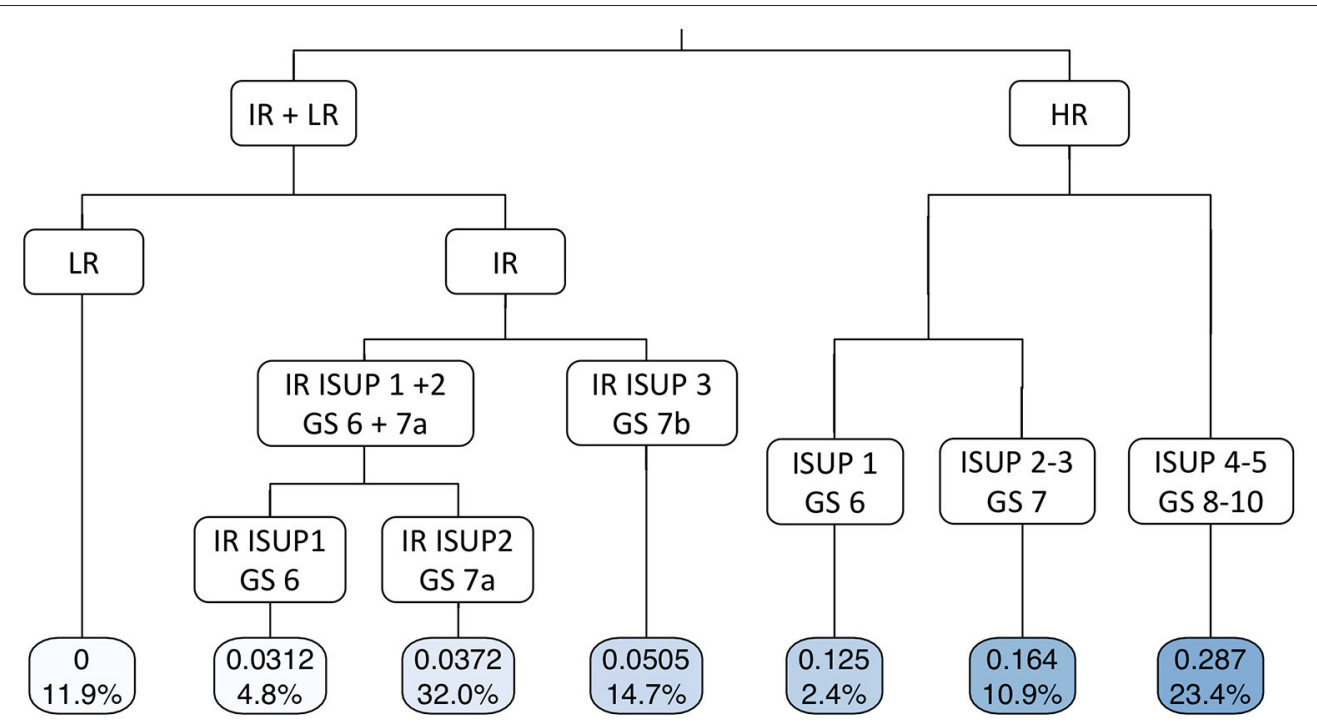

FIGURE 1 | Binary regression tree depicting probability of lymph node invasion in patients, who underwent radical prostatectomy at University Hospital Frankfurt according to D'Amico risk classification and ISUP grade/Gleason score (accuracy 0.753). The decimals in the bars are the predicted probability for lymph node invasion and the percentages the proportion of patients of the entire cohort. LR, low risk; IR, intermediate risk; HR, high risk; ISUP, Internal society of urological pathologists; GS, Gleason score.

TABLE 2 | Univariable and multivariable logistic regression model predicting lymph node invasion in patients, who underwent radical prostatectomy.

\begin{tabular}{|c|c|c|c|c|c|c|}
\hline & \multicolumn{3}{|c|}{ Univariable } & \multicolumn{3}{|c|}{ Multivariable } \\
\hline & OR & $95 \%-\mathrm{Cl}$ & $P$-value & OR & $95 \%-\mathrm{Cl}$ & $P$-value \\
\hline High risk & $\operatorname{Ref}(1.0)$ & - & - & - & - & - \\
\hline Intermediate risk GS 6 & 0.10 & $0.01-0.49$ & 0.03 & 0.12 & $0.01-0.60$ & 0.043 \\
\hline Intermediate risk GS 7a & 0.12 & $0.05-0.25$ & $<0.001$ & 0.10 & $0.04-0.22$ & $<0.001$ \\
\hline Intermediate risk GS 7b & 0.17 & $0.06-0.40$ & $<0.001$ & 0.18 & $0.06-0.44$ & $<0.001$ \\
\hline Number of removed LN & 1.04 & $1.02-1.07$ & $<0.01$ & 1.03 & $1.00-1.06$ & 0.058 \\
\hline Age & 0.99 & $0.96-1.03$ & 0.7 & 0.98 & $0.94-1.02$ & 0.4 \\
\hline Prostate volume & 1.01 & $0.99-1.02$ & 0.3 & 1.00 & $0.99-1.01$ & 0.8 \\
\hline $\mathrm{BMl}$ & 0.95 & $0.89-1.01$ & 0.13 & 0.93 & $0.86-1.00$ & 0.047 \\
\hline
\end{tabular}

GS, Gleason score; LN, lymph nodes; BMI, Body mass index; OR, Odds ratio; Cl, Confidence interval.

\section{DISCUSSIONS}

We hypothesized that differences in the rates of LNI in patients with intermediate-risk PCa may exist as patients do have variations in tumor characteristics at biopsy. Especially, the risk of LNI could be dependent on ISUP grade in patients with intermediate-risk PCa. We tested this hypothesis within our institutional RP database and arrived at several noteworthy findings.

First, we identified important differences in patient characteristics within the patient with intermediate-risk PCa subgroup. In the intermediate-risk subgroup, patients with IR-ISUP1 accounted for the fewest proportion of patients (5\%), relative to IR-ISUP2 (32\%) and IR-ISUP3 (15\%). Moreover, patients with IR-ISUP1 exhibited highest PSA $(11.5 \mathrm{ng} / \mathrm{ml})$, relative to both IR-ISUP2 and IR-ISUP3 subgroups (6.6 and $7.5 \mathrm{ng} / \mathrm{ml})$. Additionally, clinical T2b stage was predominant in IR-ISUP1 (31\%), relative to IR-ISUP2 (11\%) and IR-ISUP3 (10\%).
Those observations reflect the heterogeneity of patients with intermediate-risk $\mathrm{PCa}$ and make it crucial to distinguish several risk groups within those patients with intermediate-risk $\mathrm{PCa}$ $(16,20)$, since each of our subgroups differ in patient and tumor characteristics. For example, National Comprehensive Cancer Network (NCCN) guidelines recommend distinguishing and stratify between favorable and unfavorable intermediate-risk $\mathrm{PCa}$, taking into account, that IR-ISUP3 accounts for an unfavorable status in any case (21). The distinction into three groups according to ISUP grade in the present study leads automatically to higher proportions of PSA $>10 \mathrm{ng} / \mathrm{ml}$ and/or cT2 $\mathrm{b}$ stages in patients with IR-ISUP1, since otherwise those patients would have been classified into low-risk D'Amico group and thus reflect a relatively rare cohort of patients with intermediate-risk PCa. However, in IR-ISUP1 patients, the higher proportions of more unfavorable tumor characteristics did not translate into higher proportions of unfavorable pathological characteristics. Conversely, unfavorable pathological characteristics, for 
example, $\geq$ pT3 stage were the highest in patients with IR-ISUP3 and IR-ISUP2 PCa, in that order. This observation may confirm that ISUP grade/Gleason score is the best predictor for worse pathological outcome of those tumor characteristics used within the D'Amico classification (22-25).

Second, we showed important findings concerning the influence of ISUP grade at biopsy and risk of LNI after PLND. According to intermediate-risk stratified analyses, IRISUP3 exhibited highest rates of LNI (5\%), followed by IRISUP2 (4\%) and IR-ISUP1 (3\%), in that order. Moreover, significantly higher rates of LNI were observed in patients with high-risk PCa (24\%), where the stratification according to ISUP grades also yielded to different probabilities of LNI (Figure 1). Conversely, none of the patients with low-risk PCa exhibited LNI. Furthermore, in multivariate logistic regression models after adjustment for patient and surgical characteristics, all intermediate-risk subgroups were independently associated with lower rates of LNI compared with men with high-risk PCa. Our observations can be confirmed by previous investigations. For example, Mandel et al. investigated a LNI in 3.3\% of patients with intermediate-risk PCa harboring ISUP 1 at biopsy (19). Since European and NCCN guidelines recommend PLND in selected patients with, respectively, $>5$ and $>2 \%$ risk of LNI, those criteria would only fit to our IR-ISUP3 cohort for European and for all intermediate-risk cohorts for NCCN guidelines $(10,11)$. Nonetheless, it is noteworthy to consider that a contemporary investigation demonstrated that PLND in patients with $>5 \%$ risk of LNI did not yield to better oncological survival outcome in patients with intermediate- and high-risk $\mathrm{PCa}$, relative to non-performance of PLND (24). However, our results suggest that stratification according to ISUP grade in patients with intermediate-risk PCa predicts LNI for clinical considerations, as previous publications have also shown ISUP grade/Gleason score at biopsy in general to be an independent risk factor for LNI (26). In consequence, PLND should be performed in patients with higher ISUP/Gleason score at biopsy and might be omitted in selected patients with intermediate risk with ISUP grade 1.

Third, the median numbers of removed lymph nodes were 10 , 12, and 12 for IR-ISUP1, IR-ISUP2, and IR-ISUP3 subgroups, respectively. In univariable logistic regression, the number of removed lymph nodes was a predictor of LNI but failed statistical significance in multivariable analyses $(p=0.058)$. Since guidelines recommend an extended PLND, due to improved staging information, our median number of removed lymph nodes in intermediate-risk subgroups may suggest that extended PLND was not performed in all patients especially with IRISUP1. Nevertheless, a conclusive assessment cannot be made solely looking at the numbers of removed lymph nodes, instead of anatomical regions $(6,10,27)$. However, it should be emphasized that some studies question the need for extended PLND in

\section{REFERENCES}

1. Siegel RL, Miller KD, Jemal A. Cancer statistics 2019. CA Cancer J Clin. (2019) 69:7-34. doi: 10.3322/caac. 21551 intermediate-risk PCa and $90 \%$ of all lymph node metastases in cT2 tumors can be detected with the removal of six to eight lymph nodes $(28,29)$. In consequence, our median number of removed lymph nodes might discover the majority of lymph node metastases in our intermediate-risk PCa patient subgroups.

Our study has several limitations. First, our study is based on retrospective analyses. Second, LNI is affected by the extension of PLND and number of removed lymph nodes. Unfortunately, information regarding the field and template of the LND were not available for the current study and may also be influenced by the surgical approach. Third, information on patients' comorbidities are missing. Fourth, no information regarding the use of modern staging modalities such as PSMA PET/CT was available. Furthermore, no information of the Gleason scores and numbers of different PCa foci were available. Finally, our results consist of no data according to biochemical recurrence or survival. However, this study did not aim to investigate those outcomes.

Taken together, important differences according to LNI in different intermediate-risk subgroups exist. Intermediate-risk $\mathrm{PCa}$ is heterogeneous according to patient and tumor characteristics and can be stratified according to ISUP grade. The risk of LNI increases with higher ISUP grade at biopsy in intermediate-risk PCa and reaches $>5 \%$ only in the IR-ISUP3 subgroup. Therefore, PLND might be omitted in selected patients with intermediaterisk $\mathrm{PCa}$.

\section{DATA AVAILABILITY STATEMENT}

The raw data supporting the conclusions of this article will be made available by the authors, without undue reservation.

\section{ETHICS STATEMENT}

The studies involving human participants were reviewed and approved by Ethics Committee, Goethe University Hospital Frankfurt, SUG-1-2018. Written informed consent for participation was not required for this study in accordance with the national legislation and the institutional requirements.

\section{AUTHOR CONTRIBUTIONS}

MW: manuscript writing/editing, protocol/project development, and data analysis. $\mathrm{BH}, \mathrm{CWi}, \mathrm{CWü}, \mathrm{AB}$, and $\mathrm{PM}$ : manuscript writing/editing. MW and FP: data analysis. $\mathrm{CH}$ : data collection or management. PK: data analysis and manuscript writing/editing. FC: protocol/project development. LK: protocol/project development and manuscript writing/editing. All authors contributed to the article and approved the submitted version.

2. Arnold M, Karim-Kos HE, Coebergh JW, Byrnes G, Antilla A, Ferlay J, et al. Recent trends in incidence of five common cancers in 26 European countries since 1988: analysis of the European Cancer Observatory. Eur J Cancer. (2015) 51:1164-87. doi: 10.1016/j.ejca.2013.09.002 
3. Wenzel M, Würnschimmel C, Nocera L, Ruvolo CC, Tian Z, Saad F, et al. The effect of primary urological cancers on survival in men with secondary prostate cancer. Prostate. (2021) 81:1149-58. doi: 10.1002/pros.24209

4. Würnschimmel C, Wenzel M, Wang N, Tian Z, Karakiewicz PI, Graefen $\mathrm{M}$, et al. Radical prostatectomy for localized prostate cancer: 20-year oncological outcomes from a German high-volume center. Urol Oncol. (2021). doi: 10.1016/j.urolonc.2021.04.031

5. Wenzel M, Würnschimmel C, Ruvolo CC, Nocera L, Tian Z, Saad $\mathrm{F}$, et al. Increasing rates of NCCN high and very high-risk prostate cancer versus number of prostate biopsy cores. Prostate. (2021) 81:874-81. doi: $10.1002 /$ pros. 24184

6. Fossati N, Willemse P-PM, Van den Broeck T, van den Bergh RCN, Yuan CY, Briers E, et al. The benefits and harms of different extents of lymph node dissection during radical prostatectomy for prostate cancer: a systematic review. Eur Urol. (2017) 72:84-109. doi: 10.1016/j.eururo.2016.12.003

7. Wenzel M, Würnschimmel C, Nocera L, Collà Ruvolo C, Tian Z, Shariat SF, et al. The effect of lymph node dissection on cancer-specific survival in salvage radical prostatectomy patients. Prostate. (2021) 81:33946. doi: $10.1002 /$ pros. 24112

8. Briganti A, Chun FK-H, Salonia A, Suardi N, Gallina A, Da Pozzo LF, et al. Complications and other surgical outcomes associated with extended pelvic lymphadenectomy in men with localized prostate cancer. Eur Urol. (2006) 50:1006-13. doi: 10.1016/j.eururo.2006.08.015

9. Ploussard G, Briganti A, de la Taille A, Haese A, Heidenreich A, Menon M, et al. Pelvic lymph node dissection during robot-assisted radical prostatectomy: efficacy, limitations, and complications-a systematic review of the literature. Eur Urol. (2014) 65:7-16. doi: 10.1016/j.eururo.2013.03.057

10. Mottet N, Cornford P, Van den Bergh RCN, Briers E, Van den Broeck T, Cumberbatch MG, et al. EAU - EANM - ESTRO - ESUR - SIOG. Guidelines on Prostate Cancer. Eur Urol. (2021) 79:24362. doi: 10.1016/j.eururo.2020.09.042

11. Briganti A, Larcher A, Abdollah F, Capitanio U, Gallina A, Suardi N, et al. Updated nomogram predicting lymph node invasion in patients with prostate cancer undergoing extended pelvic lymph node dissection: the essential importance of percentage of positive cores. Eur Urol. (2012) 61:4807. doi: 10.1016/j.eururo.2011.10.044

12. Abdollah F, Cozzarini C, Suardi N, Gallina A, Capitanio U, Bianchi M, et al. Indications for pelvic nodal treatment in prostate cancer should change. Validation of the Roach formula in a large extended nodal dissection series. Int J Radiat Oncol Biol Phys. (2012) 83:624-9. doi: 10.1016/j.ijrobp.2011.06.2014

13. Dell'Oglio P, Abdollah F, Suardi N, Gallina A, Cucchiara V, Vizziello D, et al. External validation of the European association of urology recommendations for pelvic lymph node dissection in patients treated with robot-assisted radical prostatectomy. J Endourol. (2014) 28:416-23. doi: 10.1089/end.2013.0571

14. Gandaglia G, Fossati N, Zaffuto E, Bandini M, Dell'Oglio P, Bravi CA, et al. Development and internal validation of a novel model to identify the candidates for extended pelvic lymph node dissection in prostate cancer. Eur Urol. (2017) 72:632-40. doi: 10.1016/j.eururo.2017.03.049

15. Nocera L, Sood A, Dalela D, Gild P, Rogers CG, Peabody JO, et al. Rate and extent of pelvic lymph node dissection in the us prostate cancer patients treated with radical prostatectomy. Clin Genitourin Cancer. (2018) 16:e45167. doi: 10.1016/j.clgc.2017.10.003

16. Preisser F, Cooperberg MR, Crook J, Feng F, Graefen M, Karakiewicz PI, et al. Intermediate-risk prostate cancer: stratification and management. Eur Urol Oncol. (2020) 3:270-80. doi: 10.1016/j.euo.2020.03.002

17. Wenzel M, Collà Ruvolo C, Nocera L, Würnschimmel C, Tian Z, Shariat SF, et al. Regional differences in patient age and prostate cancer characteristics and rates of treatment modalities in favorable and unfavorable intermediate risk prostate cancer across United States SEER registries. Cancer Epidemiol. (2021) 74:101994. doi: 10.1016/j.canep.2021.101994

18. Wenzel M, Nocera L, Collà Ruvolo C, Würnschimmel C, Tian Z, Shariat $\mathrm{SF}$, et al. Racial/ethnic disparities in tumor characteristics and treatments in favorable and unfavorable intermediate risk prostate cancer. J Urol. (2021) 206:69-79. doi: 10.1097/JU.0000000000001695

19. Mandel P, Kriegmair MC, Veleva V, Salomon G, Graefen M, Huland H, et al. The role of pelvic lymph node dissection during radical prostatectomy in patients with gleason 6 intermediate-risk prostate cancer. Urology. (2016) 93:141-6. doi: 10.1016/j.urology.2016.02.046
20. Kane CJ, Eggener SE, Shindel AW, Andriole GL. Variability in outcomes for patients with intermediate-risk prostate cancer (Gleason Score 7, International Society of Urological Pathology Gleason Group 2-3) and implications for risk stratification: a systematic review. Eur Urol Focus. (2017) 3:487-97. doi: 10.1016/j.euf.2016.10.010

21. Mohler JL, Antonarakis ES, Armstrong AJ, D'Amico AV, Davis BJ, Dorff T, et al. Prostate cancer, version 1.2020, NCCN clinical practice guidelines in oncology. J Natl Compr Canc Netw. (2019) 17:479505. doi: 10.6004/jnccn.2019.0023

22. Zumsteg ZS, Chen Z, Howard LE, Amling CL, Aronson WJ, Cooperberg $M R$, et al. Number of unfavorable intermediate-risk factors predicts pathologic upstaging and prostate cancer-specific mortality following radical prostatectomy: results from the SEARCH database. Prostate. (2017) 77:15463. doi: $10.1002 /$ pros. 23255

23. Nocera L, Wenzel M, Collà Ruvolo C, Würnschimmel C, Tian Z, Gandaglia G, et al. The impact of race/ethnicity on upstaging and/or upgrading rates among intermediate risk prostate cancer patients treated with radical prostatectomy. World J Urol. (2021). doi: 10.1007/s00345-021-03816-0. [Epub ahead of print].

24. Wenzel M, Würnschimmel C, Chierigo F, Flammia RS, Tian Z, Shariat SF, et al. Nomogram predicting downgrading in national comprehensive cancer network high-risk prostate cancer patients treated with radical prostatectomy. Eur Urol Focus. (2021). doi: 10.1016/j.euf.2021.07.008. [Epub ahead of print].

25. Preisser F, van den Bergh RCN, Gandaglia G, Ost P, Surcel CI, Sooriakumaran P, et al. Effect of extended pelvic lymph node dissection on oncologic outcomes in patients with D'Amico intermediate and high risk prostate cancer treated with radical prostatectomy: a multiinstitutional study. J Urol. (2020) 203:338-43. doi: 10.1097/JU.00000000000 00504

26. Cao H-M, Wan Z, Wu Y, Wang H-Y, Guan C. Development and internal validation of a novel model and markers to identify the candidates for lymph node metastasis in patients with prostate cancer. Medicine (Baltimore). (2019) 98:e16534. doi: 10.1097/MD.0000000000016534

27. Mattei A, Fuechsel FG, Bhatta Dhar N, Warncke SH, Thalmann GN, Krause $\mathrm{T}$, et al. The template of the primary lymphatic landing sites of the prostate should be revisited: results of a multimodality mapping study. Eur Urol. (2008) 53:118-25. doi: 10.1016/j.eururo.2007.07.035

28. Roscigno M, Nicolai M, La Croce G, Pellucchi F, Scarcello M, Saccà A, et al. Difference in frequency and distribution of nodal metastases between intermediate and high risk prostate cancer patients: results of a superextended pelvic lymph node dissection. Front Surg. (2018) 5:52. doi: 10.3389/fsurg.2018.00052

29. Kluth LA, Abdollah F, Xylinas E, Rieken M, Fajkovic H, Seitz C, et al. Clinical nodal staging scores for prostate cancer: a proposal for preoperative risk assessment. Br J Cancer. (2014) 111:213-9. doi: 10.1038/bjc.20 14.311

Conflict of Interest: The authors declare that the research was conducted in the absence of any commercial or financial relationships that could be construed as a potential conflict of interest.

The reviewer GD declared a past co-authorship with one of the authors CWü to the handling editor.

Publisher's Note: All claims expressed in this article are solely those of the authors and do not necessarily represent those of their affiliated organizations, or those of the publisher, the editors and the reviewers. Any product that may be evaluated in this article, or claim that may be made by its manufacturer, is not guaranteed or endorsed by the publisher.

Copyright (C) 2021 Wenzel, Preisser, Hoeh, Welte, Humke, Wittler, Würnschimmel, Becker, Karakiewicz, Chun, Mandel and Kluth. This is an open-access article distributed under the terms of the Creative Commons Attribution License (CC BY). The use, distribution or reproduction in other forums is permitted, provided the original author(s) and the copyright owner(s) are credited and that the original publication in this journal is cited, in accordance with accepted academic practice. No use, distribution or reproduction is permitted which does not comply with these terms. 